\title{
Pathology of the Fallopian Tube: Tubal Involvement by Ovarian Tumors and Incidental Findings in the Nontumoral Setting
}

\author{
Georgia Karpathiou $^{\mathrm{a}}$ Celine Chauleur $^{\mathrm{b}}$ Melany Venet $^{\mathrm{a}}$ Alix Clemenson $^{\mathrm{a}}$ \\ Michel Peoc' $h^{a}$ \\ ${ }^{a}$ Department of Pathology, North Hospital, University Hospital of Saint-Etienne, Saint-Etienne, France; ${ }^{\text {b Department }}$ \\ of Obstetrics and Gynecology, North Hospital, University Hospital of Saint-Etienne, Saint-Etienne, France
}

\section{Keywords}

STIC · Adenofibroma - Transitional metaplasia •

Tubal-peritoneal junction · Tubal invasion · Nonserous tumors · Endometriosis · Mucinous metaplasia

\begin{abstract}
The fallopian tube is thought to be the site of origin of most high-grade serous carcinomas (HGSCs). However, how often the tube is abnormal in the setting of other ovarian tumors is unknown. The aim of this study is to define the frequency of tubal abnormalities in the tumoral $(n=245)$ and nontumoral ( $n=184$ ) setting. We found that in ovarian tumors, $52.2 \%$ of the tubes were normal, while $39.2 \%$ were affected by the tumor. Abnormal tubes were found in $80 \%$ of HGSCs, in $21 \%$ of mucinous carcinomas, in $83.3 \%$ of seromucinous carcinomas, in $33.3 \%$ of endometrioid carcinomas, in $20 \%$ of clear-cell carcinomas, and in $10.5 \%$ of borderline tumors. Among normal tubes, almost $70 \%$ were histologically normal; transitional metaplasia was present in $17.4 \%$, endometriosis in $8.1 \%$, and adenofibroma in $2.2 \%$, and $1.1 \%$ had an incidental serous intraepithelial tubal carcinoma. To conclude, the fallopian tube is abnormal in most serous carcinomas, and in a smaller number of endometrioid, clear-cell and mucinous carcinomas as well as borderline tumors. It is often abnormal in seromucinous tumors, but larger series are needed to study this rare subtype.
\end{abstract}

(c) 2020 S. Karger AG, Basel

\section{Introduction}

The histogenesis of ovarian epithelial tumors has always been a problematic issue due to the absence of a normal counterpart inside the ovary; thus, a mesothelial origin was the principal theory for decades. Almost two decades ago, Dubeau [1] proposed a nonovarian origin for these tumors, with the secondary Müllerian system being a possible site of origin. Since some studies showed frequent findings of occult carcinomas in fallopian tubes prophylactically removed in $B R C A$ mutation carriers [2], the fallopian tube has become the preferred site of origin of pelvic high-grade serous carcinomas (HGSCs). Thus, studies examining fallopian tubes for the presence of serous intraepithelial tubal carcinoma (STIC) revealed that almost $60 \%$ of HGSCs contained STIC foci $[3,4]$. However, the tubes are often heavily invaded or even completely absent in HGSC, impeding their true evaluation. Furthermore, despite several studies examining the frequency of tubal abnormalities in the setting of HGSC, the tube status in the setting of other ovarian tumors has not been evaluated. Thus, the aim of this study is to define the frequency of tubal pathology in the setting of ovarian tumor. 

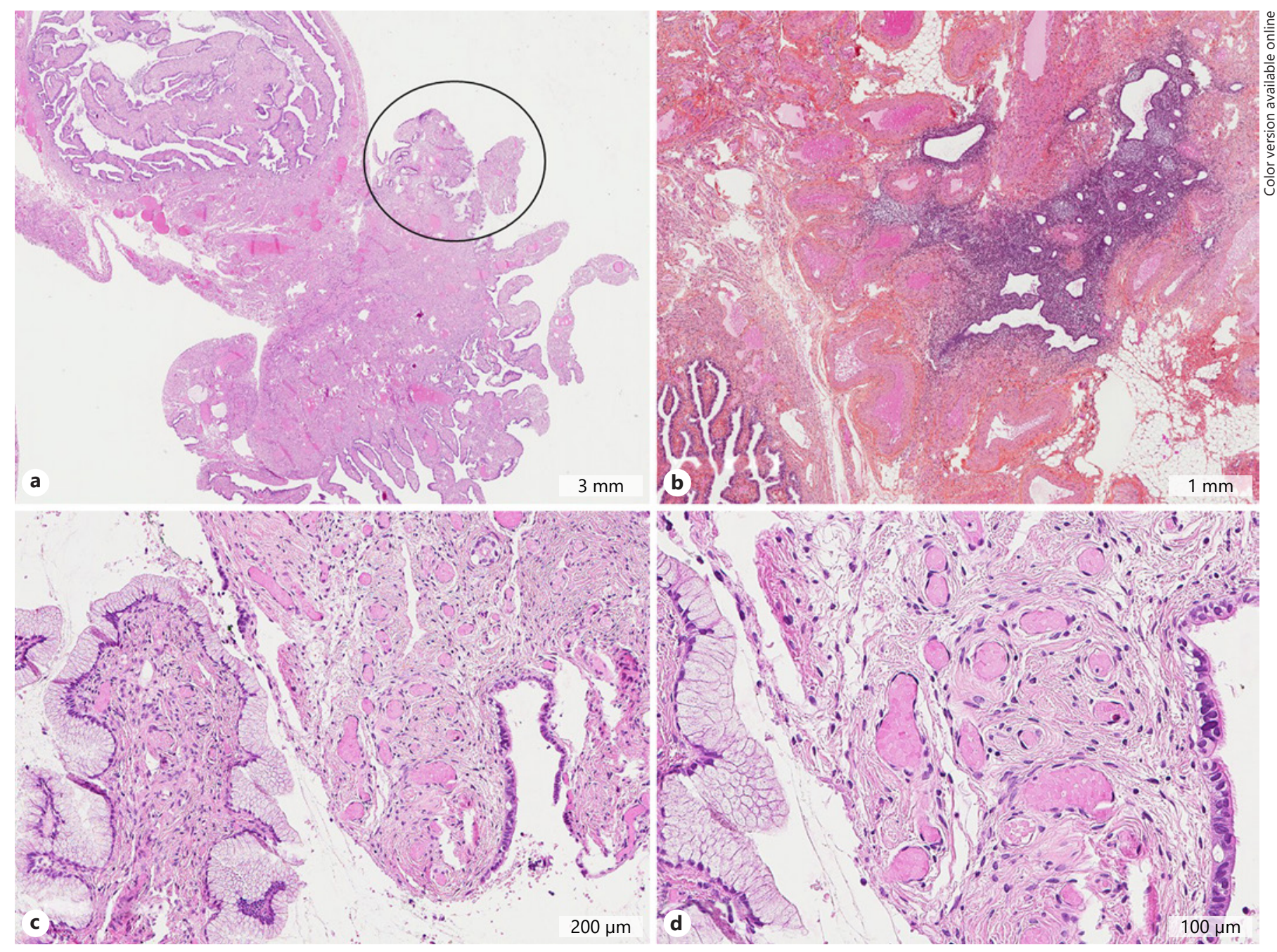

Fig. 1. a Mucinous metaplasia of the fallopian tube, a finding that could be associated with Peutz-Jeghers syndrome (hematoxylin, eosin, saffran $[\mathrm{HES}] \times 7$ ). $\mathbf{b}$ Endometriosis of the fallopian tube $(\mathrm{HES} \times 20)$. c, $\mathbf{d}$ Mucinous metaplasia at higher magnification $(\mathbf{c} \times 100 ; \mathbf{d} \times 200)$, showing metaplastic mucinous epithelium on the left and normal ciliated epithelium on the right.

\section{Materials and Methods}

\section{Study Population}

The study included a group of patients treated for an ovarian tumor (ovarian group) and a group of patients with clinically normal tubes (control group). The first group comprised patients diagnosed with an ovarian borderline or malignant tumor, treated surgically without neoadjuvant chemotherapy. Cases with a certain tubal origin (tubal tumors with microscopically normal or minimally infiltrated ovaries) or certain peritoneal origin (peritoneal tumors with normal tubes/ovaries) were originally excluded, as well as patients with a previous history of tubal excision.

The control group comprised patients who had tubes removed for a uterine or benign ovarian/tubal pathology. Patients with ectopic pregnancy or salpingitis/hydrosalpinx were originally excluded from the study.

\section{Histopathological Evaluation}

All available slides were histopathologically evaluated. The following lesions were recorded: STIC, endometriosis, transitional metaplasia, adenofibroma, and mucinous metaplasia (Fig. 1). All cases were prospectively and routinely studied by the SEE-FIM protocol. Furthermore, in the ovarian group, abnormal tube status in regard to the tumor was noted as follows: (a) invasion by the tumor, defined as whole tubal wall involvement; (b) tube completely unidentifiable and presumably entirely incorporated into the mass; and (c) tube only focally identifiable but not infiltrated in this particular part, and here we included cases where only a small portion of a normal tube was identified, the rest being presumably incorporated into the mass [5]. When one of these abnormalities was found, the fallopian tube was defined as abnormal concerning the tumor. 
Table 1. Group characteristics

\begin{tabular}{|c|c|}
\hline Characteristics & $n(\%)$ \\
\hline \multicolumn{2}{|l|}{ Surgery cause in the control group $(n=184)$} \\
\hline Leiomyoma-adenomyosis & $46(25)$ \\
\hline Prolapsus & $7(3.8)$ \\
\hline Endometrial carcinoma or hyperplasia with atypia & $42(22.8)$ \\
\hline Cervical carcinoma/dysplasia & $14(7.6)$ \\
\hline Benign ovarian tumor (teratoma, fibroma/thecoma, cystadenoma) & $66(35.9)$ \\
\hline Prophylactic annexectomy (BRCA mutation or familial/personal history) & $4(2.2)$ \\
\hline Sterilization & $5(2.7)$ \\
\hline \multicolumn{2}{|l|}{ Ovarian tumors $(n=245)$} \\
\hline Serous borderline & $48(19.6)$ \\
\hline Mucinous borderline & $40(16.3)$ \\
\hline Seromucinous borderline & $5(2)$ \\
\hline Clear-cell borderline & $1(0.4)$ \\
\hline Brenner borderline & $1(0.4)$ \\
\hline High-grade serous carcinoma & $90(36.7)$ \\
\hline Low-grade serous carcinoma & $2(0.8)$ \\
\hline Mucinous carcinoma & $19(7.8)$ \\
\hline Seromucinous carcinoma & $6(2.5)$ \\
\hline Endometrioid carcinoma & $18(7.4)$ \\
\hline Clear-cell carcinoma & $15(6.1)$ \\
\hline \multicolumn{2}{|l|}{ Tubal assessment in the control group $(n=184)$} \\
\hline Normal & $128(69.5)$ \\
\hline STIC & $4(2.2)$ \\
\hline Adenofibroma & $4(2.2)$ \\
\hline Transitional metaplasia & $32(17.4)$ \\
\hline Endometriosis & $15(8.1)$ \\
\hline Mucinous metaplasia & $1(0.5)$ \\
\hline \multicolumn{2}{|l|}{ Tubal assessment in the ovarian group $(n=245)$} \\
\hline Normal & $128(52.2)$ \\
\hline STIC & $3(1.2)$ \\
\hline Infiltrated by ovarian tumor & $42(17.2)$ \\
\hline Only focally visible, but not infiltrated in this part & $11(4.4)$ \\
\hline Absent & $43(17.6)$ \\
\hline Transitional metaplasia & $2(0.8)$ \\
\hline Endometriosis & $16(6.5)$ \\
\hline
\end{tabular}

Statistical Analysis

Data were analyzed using StatView software (Abacus Concepts, Berkeley, CA, USA). Relationships between 2 groups were investigated using the $\chi^{2}$ test for categorical data. For all analyses, statistical significance was indicated at $p<0.05$.

\section{Results}

Tubal Assessment in the Control Group $(n=184)$

Group characteristics are shown in Table 1. Most clinically normal fallopian tubes were also histologically normal. Transitional metaplasia of the tubal-mesothelial junction was present in $17.4 \%$ of the tubes, and in another $2.2 \%$, an adenofibroma was observed (Fig. 2). There was 1 case of mucinous metaplasia $(0.5 \%)$. Genetic counseling was initially recommended given the association with Peutz-Jeghers syndrome, but due to the absence of any personal or familial history, no further actions were taken. According to her medical record, the patient did not develop any further diseases at the 2-year follow-up. Endometriosis was found in 15 cases (8.1\%). Four STICs (2.2\%) were found, confirmed by p53 and MIB1 staining. Among them, 1 patient had a family history of ovarian cancer, but with unknown BRCA status. Another patient 

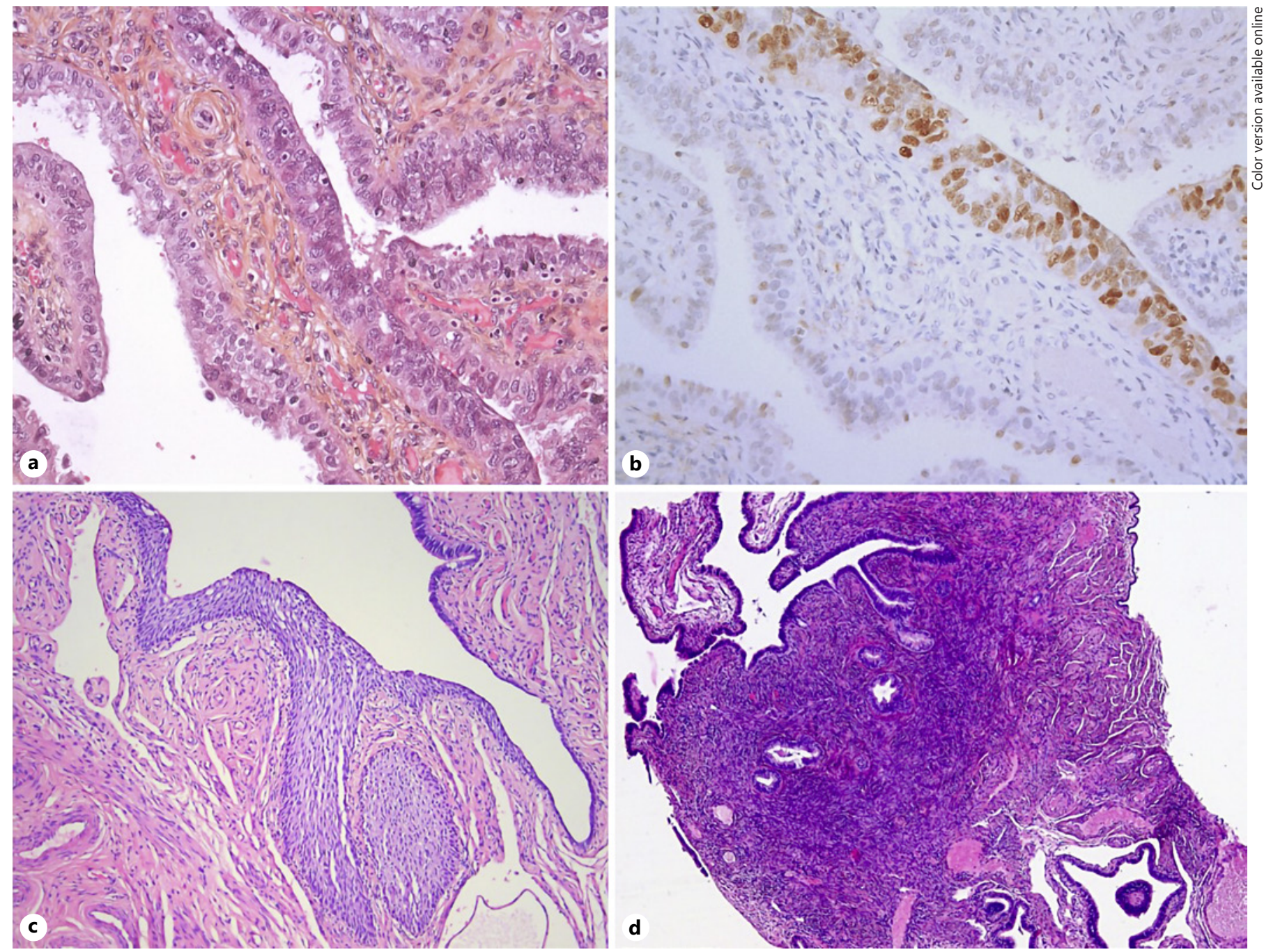

Fig. 2. a STIC in a patient with MSI $($ HES $\times 200)$. b Same case, showing heterogeneous but stronger than nearby normal epithelium p53 expression $(\times 200)$. c Transitional metaplasia of fimbrial end is found in almost $20 \%$ of normal tubes $($ HES $\times 100)$. d Tubal adenofibroma $($ HES $\times 50)$. MSI, microsatellite instability.

was operated on due to a lower uterine segment serous carcinoma infiltrating the cervix and the isthmus. It was human papillomavirus (HPV)-negative and showed microsatellite instability (MSI; loss of MSH2/PMS2 immunohistochemical expression and MSI testing with modification in 3 of the 5 markers). The STIC focus was not considered metastatic as it was limited to the mucosa, not accompanied by floating fragments inside the lumen, and WT1+, while the cervical tumor was WT1-. No MSI analysis could be performed on this focus, as the material was too limited. The other 2 patients (1.1\%) had unremarkable familial/personal clinical history. All STIC foci were found in the fimbrial end. None of these patients were operated on in the context of BRCA prophylactic salpingectomy. Intraepithelial carcinomas, adenofibromas, and mucinous metaplasia were unilateral, whereas 10 of the 32 cases of transitional metaplasia and 4 of the 15 endometriosis cases were bilateral.

Tubal Assessment in the Ovarian Group ( $n=245)$

In total, 128 (52.2\%) of the tubes excised in the setting of an ovarian tumor were histologically normal (Table 2); $96(39.2 \%)$ were either infiltrated by the tumor or completely absent or only focally identifiable (Fig. 3). Of the 96 fallopian tubes affected by the ovarian tumor, 70 (72.9\%) were HGSCs, 3 (3.1\%) were mucinous carcino- 
Table 2. Fallopian tube assessment

\begin{tabular}{|c|c|c|c|c|c|c|c|c|c|}
\hline Normal & 128 & 16 & 15 & 0 & 8 & 8 & 1 & 80 & \multirow[t]{9}{*}{$<0.0001$} \\
\hline Adenofibroma & 4 & 0 & 0 & 0 & 0 & 0 & 0 & 0 & \\
\hline Transitional metaplasia & 32 & 0 & 1 & 0 & 0 & 0 & 0 & $1 \mathrm{M}$ & \\
\hline Endometriosis & 15 & 2 & 0 & 1 & 4 & 4 & 0 & $\begin{array}{l}5 \text { (1 CC, } 2 \mathrm{M}, \\
1 \mathrm{SM}, 1 \mathrm{~S})\end{array}$ & \\
\hline Mucinous metaplasia & 1 & 0 & 0 & 0 & 0 & 0 & 0 & 0 & \\
\hline Tubal abnormality regarding the tumor & NA & 70 & 3 & 4 & 6 & 3 & 1 & 9 & \\
\hline Infiltrated & NA & 37 & 0 & 1 & 3 & 1 & 0 & 0 & \\
\hline Absent & NA & 22 & 3 & 3 & 3 & 2 & 1 & $9(2 \mathrm{SM}, 7 \mathrm{~S})$ & \\
\hline Focally visible & NA & 11 & 0 & 0 & 0 & 0 & 0 & 0 & \\
\hline
\end{tabular}

HGSC, high-grade serous carcinoma; MC, mucinous carcinoma; SMC, seromucinous carcinoma; EnC, endometrioid carcinoma; CC, clear-cell carcinoma; LGSC, low-grade serous carcinoma; M, mucinous; SM, seromucinous; S, serous. See text for details.

mas, 4 (4.2\%) were seromucinous carcinomas, 3 (3.1\%) were clear-cell carcinomas, 6 (6.3\%) were endometrioid carcinomas, 1 was a low-grade serous carcinoma, and 9 (9.4\%) were in the borderline category (serous and seromucinous). Three cases of STIC were found, 2 with an HGSC and 1 with a seromucinous carcinoma, all showing the typical morphological and immunohistochemical aspects of a STIC and all ipsilateral with the tumor. Two cases were situated in the fimbrial end and 1 in a central tubal location. Another 2 cases, both with a mucinous neoplasm, presented transitional metaplasia. Endometriosis was found in 16 cases (6.5\%); 5 cases were associated with clear-cell neoplasms (4 carcinomas, 1 borderline), 4 cases with endometrioid carcinomas, 2 with seromucinous tumors ( 1 carcinoma, 1 borderline), 3 serous ( 1 borderline, 2 carcinomas), and 2 with mucinous borderline tumors.

\section{Discussion}

In the current study, we show that almost $40 \%$ of fallopian tubes were infiltrated by an ovarian tumor, but with different frequency according to the various tumor types. This not only adds to the knowledge regarding ovarian tumor pathophysiology, but also highlights that in at least $40 \%$ of the tumors, there will be staging up of the disease, if not already advanced, due to tube involvement. Furthermore, the presence of STIC in the normal setting highlights the importance of always sampling at least the entire fimbrial end.

Fallopian Tube Pathology
Despite the fallopian tube being the target of numerous studies after the discovery of its central role in HGSC, its status in nonserous tumors has not been largely investigated. The criteria proposed to assign a tubal origin for HGSC are as follows: the presence of STIC and invasive mucosal carcinoma in the tube, or the tube partially or entirely incorporated into a tubo-ovarian mass [5]. Using the same criteria as evidence of abnormal tubes, we show that ovarian tumors accompanied by abnormal fallopian tubes are most often serous and seromucinous carcinomas, with 80 and $83.3 \%$ of cases, respectively, having an abnormal tube. This is in accordance with a report showing that almost $76 \%$ of HGSCs can be considered as tubal primaries [5]. In that study, almost half of the cases were specimens from patients treated with neoadjuvant chemotherapy and all cases (tubal/ovarian/peritoneal) were included [5]. In our study, we chose surgical specimens without neoadjuvant chemotherapy, looking clinically "ovarian." Our results are similar, thus confirming that almost $80 \%$ of "ovarian" HGSCs are of tubal origin. Furthermore, in line with the aforementioned study, we found that in most of these tubal tumors, STIC was not frequent, a finding explained by the fact that the fallopian tube had often disappeared inside the tumor.

Seromucinous carcinomas are rare, with scant data in the literature [6]. Seromucinous borderline tumors are considered to originate from foci of endometriosis, since endometriosis is present in about one-third of these tumors, and they also show ARID1A expression, as endometrioid/clear cell tumors do [7]. Despite there being only a few seromucinous carcinomas present in our se- 

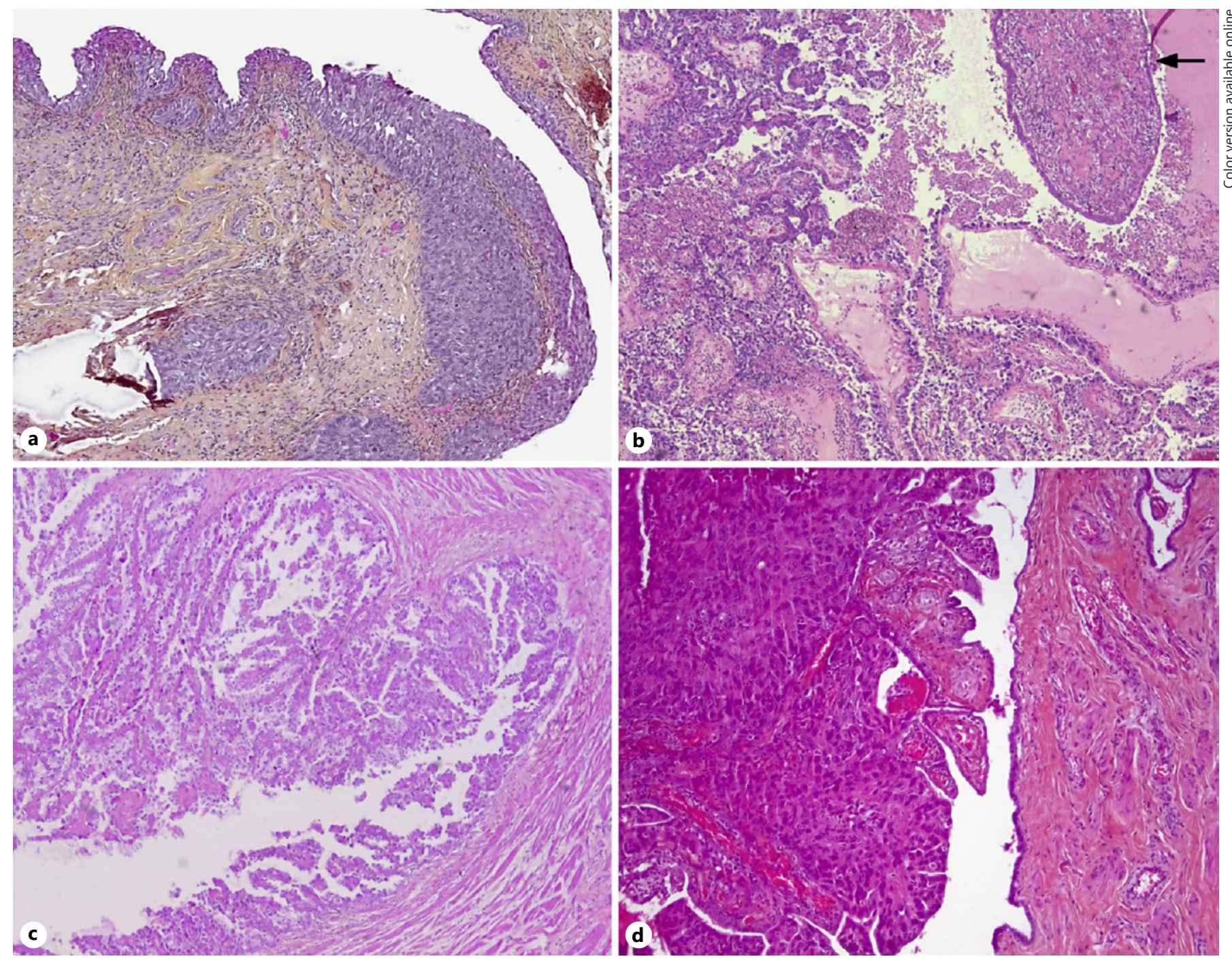

Fig. 3. a Tubal invasion in HGSC. Only the muscular layer of the tube is recognized $(\mathrm{HES} \times 50)$. b Similarly in seromucinous carcinoma, residual normal fimbrium (arrow) is seen $(\mathrm{HES} \times 50)$. c Tubal invasion in clear-cell carcinoma $(\mathrm{HES} \times 50)$. d Similarly in endometrioid carcinoma, normal mucosa is shown on the right $(\mathrm{HES} \times 100)$. HGSC, high-grade serous carcinoma.

ries, we found that most of them showed an abnormal tube, an interesting finding given their uncertain histogenesis. One case was also associated with a focus of STIC, but notably, the seromucinous carcinoma was WT1- and with normal p53 expression. For these cases of abnormal tubes in the setting of a seromucinous neoplasm, it cannot be determined if the tubal invasion is an incidental finding of tumor extension or if the tube is actually associated with the histogenesis of the tumor, and larger series to study this rare subtype are certainly needed.

Serous borderline ovarian tumors and low-grade serous carcinomas that arise from them are considered to originate from tubal cells implanted in the ovary, forming inclusion cysts [8]. We show that in $10 \%$ of borderline tumors, all serous and seromucinous, the tube was unidentifiable, providing further evidence for the possible role of the salpinx in their histogenesis. Furthermore, otherwise normal tubes may contain adenofibromas, morphologically similar to ovarian adenofibromas, another argument for the tube's participation in the pathogenesis of ovarian tumors [9].

The pathogenesis of mucinous tumors is unknown, but there are data suggesting an origin from Brenner tumors and, thus, from tubal transitional metaplasia [10]. 
Transitional metaplasia of the tubal-mesothelial junction is occasionally described in textbooks as an extremely rare lesion, based on the initial observation of 3 such cases [11]. However, we show that it was present in almost $20 \%$ of the tubes excised. This is in line with 2 other studies $[12,13]$. The importance of recognizing this lesion is to not confuse it with a STIC, from which it differs, since there is no "serous" morphology or atypia, or p53 overexpression or increased MIB1 expression. Also, detecting this metaplasia so frequently makes it an almost normal variation, and it may not be associated with tumor development. On the contrary, only very rarely this metaplasia was found in the tumoral setting, probably because it was difficult to identify due to tubal invasion. It was seen in only 2 cases, both with mucinous tumors.

As for endometrioid and clear-cell carcinomas, they are thought to arise from foci of endometriosis. Indeed, most cases with tubal endometriosis were found with these types of tumors. We also show that tubes were abnormal in almost one-third of these carcinomas; thus, an original tubal localization in the context of tubal endometriosis is possible for these cases. This is in line with the reported $81 \%$ occurrence of endometrioid carcinoma as a dominant ovarian mass and a $19 \%$ presentation with tubal invasion [4].

The presence of STIC in the nonprophylactic incidental setting has been recently described, with a reported incidence of almost 1\% [14]. We also found 2 such cases $(1.1 \%)$ in patients with no personal or familial suspicion of BRCA mutation, highlighting that the fimbrial end must always be examined. Furthermore, we found 1 case of STIC in a patient with MSI harboring a lower uterine segment serous carcinoma. Ovarian tumors typically associated with Lynch syndrome are endometrioid and clear-cell carcinomas [15-17]; endometrial tumors are most often of the endometrioid type, and serous or other histological types have been rarely reported [18, 19]. The prevalence of Lynch syndrome is also much greater in lower uterine segment tumors [20]. Our patient had a serous carcinoma involving the isthmus and the uterine cervix, negative for HPV and showing MSI. The development of an intraepithelial tubal carcinoma in this patient could be incidental or indeed associated with the instability of microsatellites, and further studies looking at such a predisposition are needed, as no data are available.

To conclude, we showed that fallopian tubes are abnormal in the majority of serous and seromucinous carcinomas and in a small amount of serous/seromucinous borderline tumors, providing further evidence for implicating the salpinx in their pathophysiology, though only a few cases of the seromucinous category were seen. Also, some mucinous, endometrioid, and clear-cell carcinomas show tubal involvement. Almost $1 \%$ of "normal" tubes harbor an intraepithelial carcinoma. Finally, transitional metaplasia of the fimbrial end is a rather frequent lesion, not to be confused with STIC.

\section{Acknowledgments}

The authors would like to thank Philippe Cosmo from the Tumorothèque/Centre de Ressources Biologiques de CHU Saint-Etienne (BRIF No. BB-0033-00041) for his assistance.

\section{Statement of Ethics}

This study was conducted according to the internationally accepted standards for research practice and reporting and approved by the IRB (DC-2015-2489).

\section{Disclosure Statement}

The authors have no conflicts of interest to declare.

\section{Author Contributions}

G.K. and M.P.: conception and design of the study; G.K., C.C., M.V., and A.C.: acquisition and analysis of data; G.K.: drafting of the manuscript; all authors approved the final version.

\footnotetext{
References

1 Dubeau L. The cell of origin of ovarian epithelial tumors and the ovarian surface epithelium dogma: does the emperor have no clothes? Gynecol Oncol. 1999 Mar;72(3):437-42.

2 Colgan TJ, Murphy J, Cole DE, Narod S, Rosen B. Occult carcinoma in prophylactic oophorectomy specimens: prevalence and association with BRCA germline mutation status. Am J Surg Pathol. 2001 Oct;25(10):1283-

3 Przybycin CG, Kurman RJ, Ronnett BM, Shih IM, Vang R. Are all pelvic (nonuterine) serous carcinomas of tubal origin? Am J Surg Pathol. 2010 Oct;34(10):1407-16.

4 Roh MH, Kindelberger D, Crum CP. Serous tubal intraepithelial carcinoma and the dominant ovarian mass: clues to serous tumor origin? Am J Surg Pathol. 2009 Mar;33(3):37683.
} 
5 Singh N, Gilks CB, Wilkinson N, McCluggage WG. Assessment of a new system for primary site assignment in high-grade serous carcinoma of the fallopian tube, ovary, and peritoneum. Histopathology. 2015 Sep;67(3):3317.

6 Karpathiou G, Chauleur C, Corsini T, Venet M, Habougit C, Honeyman F, et al. Seromucinous ovarian tumor A comparison with the rest of ovarian epithelial tumors. Ann Diagn Pathol. 2017 Apr;27:28-33.

7 Kurman RJ, Shih IM. Seromucinous Tumors of the Ovary. What's in a Name? Int J Gynecol Pathol. 2016 Jan;35(1):78-81.

8 Li J, Abushahin N, Pang S, Xiang L, Chambers SK, Fadare O, et al. Tubal origin of 'ovarian' low-grade serous carcinoma. Mod Pathol. 2011 Nov;24(11):1488-99.

9 Bossuyt V, Medeiros F, Drapkin R, Folkins AK, Crum CP, Nucci MR. Adenofibroma of the fimbria: a common entity that is indistinguishable from ovarian adenofibroma. Int J Gynecol Pathol. 2008 Jul;27(3):390-7.

10 Karpathiou G, Venet M, Mobarki M, Forest F, Chauleur C, Peoc'h M. FOXA1 is expressed in ovarian mucinous neoplasms. Pathology. 2017 Apr;49(3):271-6.
11 Egan AJ, Russell P. Transitional (urothelial) cell metaplasia of the fallopian tube mucosa: morphological assessment of three cases. Int J Gynecol Pathol. 1996 Jan;15(1):72-6.

12 Rabban JT, Crawford B, Chen LM, Powell CB, Zaloudek CJ. Transitional cell metaplasia of fallopian tube fimbriae: a potential mimic of early tubal carcinoma in risk reduction salpingo-oophorectomies from women With BRCA mutations. Am J Surg Pathol. 2009 Jan; 33(1):111-9.

13 Seidman JD, Yemelyanova A, Zaino RJ, Kurman RJ. The fallopian tube-peritoneal junction: a potential site of carcinogenesis. Int $J$ Gynecol Pathol. 2011 Jan;30(1):4-11.

14 Rabban JT, Garg K, Crawford B, Chen LM, Zaloudek CJ. Early detection of high-grade tubal serous carcinoma in women at low risk for hereditary breast and ovarian cancer syndrome by systematic examination of fallopian tubes incidentally removed during benign surgery. Am J Surg Pathol. 2014 Jun;38(6): $729-42$.

15 Karpathiou G, Batistatou A, Forest F, Clemenson A, Peoc'h M. Basic Molecular Pathology and Cytogenetics for Practicing Pathologists: Correlation With Morphology and With a Focus on Aspects of Diagnostic or Therapeutic Utility. Adv Anat Pathol. 2016 Nov;23(6):368-80.
16 Chui MH, Ryan P, Radigan J, Ferguson SE Pollett A, Aronson M, et al. The histomorphology of Lynch syndrome-associated ovarian carcinomas: toward a subtype-specific screening strategy. Am J Surg Pathol. 2014 Sep;38(9):1173-81.

17 Jensen KC, Mariappan MR, Putcha GV, Husain A, Chun N, Ford JM, et al. Microsatellite instability and mismatch repair protein defects in ovarian epithelial neoplasms in patients 50 years of age and younger. Am J Surg Pathol. 2008 Jul;32(7):1029-37.

18 Mills AM, Liou S, Ford JM, Berek JS, Pai RK, Longacre TA. Lynch syndrome screening should be considered for all patients with newly diagnosed endometrial cancer. Am J Surg Pathol. 2014 Nov;38(11):1501-9.

19 Ryan P, Mulligan AM, Aronson M, Ferguson SE, Bapat B, Semotiuk K, et al. Comparison of clinical schemas and morphologic features in predicting Lynch syndrome in mutation-positive patients with endometrial cancer encountered in the context of familial gastrointestinal cancer registries. Cancer. $2012 \mathrm{Feb}$; 118(3):681-8.

20 Westin SN, Lacour RA, Urbauer DL, Luthra $\mathrm{R}$, Bodurka DC, Lu KH, et al. Carcinoma of the lower uterine segment: a newly described association with Lynch syndrome. J Clin Oncol. 2008 Dec;26(36):5965-71. 